\title{
Modeling of Bias Dependent Fluctuations of Flicker Noise of MOSFETs
}

\author{
Ken'ichiro Sonoda, Motoaki Tanizawa, Katsumi Eikyu, Kiyoshi Ishikawa, \\ Toshio Kumamoto*, Hiroyuki Kouno*, and Masahide Inuishi \\ ULSI Development Center, System LSI Div.*, \\ Mitsubishi Electric Corp., Hyogo 664-8641, Japan \\ sonoda@lsi.melco.co.jp
}

\begin{abstract}
Drain and gate bias dependent fluctuations of flicker noise of MOSFETs are explained in terms of carrier concentration distributions in a MOSFET channel. A proposed model well describes the increase of the fluctuation in the saturation region of operation. In addition, the gate bias dependence of the fluctuation in the saturation region can also be calculated using the model. Our model predicts that for any gate voltage change, the fluctuation in the saturation region will be $2-2.5$ times that in the linear region.
\end{abstract}

\section{Introduction}

A typical noise spectrum of MOSFETs exhibits $1 / f$ dependence below $1 \mathrm{MHz}$, so that noise characteristics of CMOS circuits in the audio frequency range is determined by the flicker noise. Moreover, RF characteristics such as noise figure and oscillator phase noise are affected by the flicker noise through upconversion of low frequency noise (Hajimiri and Lee 1998). For RF application, long channel or large gate area options, which are usually adopted for low noise application in low frequency range, are no longer acceptable because of low cut-off frequency and large parasitic capacitance. As the gate area shrinks, the number of interface traps in the area decreases and the relative fluctuation in that number becomes remarkable, which leads to an increase in the variation of flicker noise from sample to sample. In a previous work (Brederlow et al. 1999), the fluctuation is expressed as a function of $V_{\mathrm{ds}} /\left(V_{\mathrm{gs}}-V_{\mathrm{th}}\right)$ in the linear region and is independent of bias in saturation region. However, our experimental results show that the fluctuation depend on gate bias even in the saturation condition. In this paper, a model is proposed to explain the fluctuation in both linear and saturation regions, and the gate voltage dependence of the fluctuation.

\section{Measurement}

A setup for flicker noise measurement is schematically shown in Fig. 1. A DUT is biased to form a source-follower amplifier. Measured noise voltage, $v_{0}$, is divided by the voltage gain of the amplifier to give input referred noise voltage. The gain can be measured with the same setup, except for a smallsignal AC voltage source which is attached to the gate of the DUT. The output 
voltage of this configuration is insensitive to power supply noise because the gate electrode, which is the most sensitive to the power supply noise, is tied directly to the ground. In common-source configuration, on the contrary, the noise voltage applied to the gate electrode is amplified by $g_{\mathrm{m}}$-times and appears at the output, which may mask the flicker noise signal to be measured.

An example of measured input referred noise voltage spectrum $S_{V} \equiv S_{I} / g_{\mathrm{m}}^{2}$ is shown in Fig. 2. The fluctuation of the noise spectrum clearly depends on bias conditions, which will be discussed in the next section. From our measurement results, the fluctuation of $g_{\mathrm{m}}$ is much smaller than that of $S_{V}$ for all bias conditions, so that the relative fluctuations of $S_{V}$ can be regarded as the same as those of $S_{I}$.

\section{Fluctuation Modeling}

The capture-emission process induces a fluctuation in the number of carriers and a correlated mobility variation, which is called the correlated numbermobility fluctuation $(\Delta N-\Delta \mu)$ model, on which the BSIM3 flicker noise model is based. According to the $\Delta N-\Delta \mu$ model, the flicker noise current power spectrum, $S_{I}$, at frequency $f$ is given by (Hung et al. 1990)

$$
S_{I}=\frac{I^{2}}{f L_{\text {eff }}} \int_{0}^{L_{\text {eff }}} \eta d x
$$

where $\eta \equiv\left(1 / L_{\text {eff }} W_{\text {eff }}\right)(1 / n+\alpha \mu)^{2} N_{\mathrm{t}} k T / \gamma, I$ is the drain current, $n$ is the carrier density per unit area, $N_{\mathrm{t}}$ is the trap density per unit volume and energy, and $\gamma$ is the attenuation coefficient of the electron wave function in the oxide. Measured input referred noise is shown in Fig. 3 with simulation results of $\Delta N$ $\Delta \mu$ (Eq. (1)), SPICE2 $\left(S_{I}=\mathrm{KF} I_{\mathrm{d}}^{\mathrm{AF}} / C_{\mathrm{ox}} L_{\text {eff }}^{2} f^{\mathrm{EF}}\right.$ ), and Gray-Meyer (Gray and Meyer 1993) $\left(S_{I}=\mathrm{KF} g_{\mathrm{m}}^{2} / C_{\mathrm{ox}} W_{\mathrm{eff}} L_{\mathrm{eff}} f^{\mathrm{EF}}\right)$ models. The $\Delta N-\Delta \mu$ model with the mobility fluctuation parameter $\alpha=0$ well describes the gate voltage dependence of $S_{V}$, which means the mobility fluctuation is negligible for our samples. The other two models cannot accurately predict the bias dependence. Note that the increase of $S_{V}$ with $V_{\mathrm{gs}}$ comes from the gate voltage dependence of $g_{\mathrm{m}}$, or $\mu$ (Ziel and Park 1979).

The gate area dependence of $S_{V}$ and its standard deviation is shown in Fig. 4. The noise spectrum $S_{V}$ is inversely proportional to the gate area, $W_{\text {eff }} L_{\text {eff }}$. The normalized standard deviation is proportional to $1 /\left(W_{\mathrm{eff}} L_{\mathrm{eff}}\right)^{1 / 2}$ as expected from Pelgrom's rule (Pelgrom et al. 1989).

The drain bias dependence of the noise fluctuation is shown in Fig. 5. As the drain voltage increases, the fluctuation increases and saturates. The saturation level depends on the gate voltage, as shown in Fig. 6. The bias dependence of the noise is modeled as

$$
\frac{\sigma\left(S_{V}\right)}{S_{V}}=\left(\frac{\sqrt{\frac{1}{L_{\text {eff }}} \int_{0}^{L_{\text {eff }}} \eta^{2} d x}}{\frac{1}{L_{\text {eff }}} \int_{0}^{L_{\text {eff }}} \eta d x}\right)\left(\frac{1}{\sqrt{N_{\mathrm{t}} k T L_{\text {eff }} W_{\text {eff }} / \gamma}}\right) .
$$

In the linear region limit of $V_{\mathrm{ds}} \rightarrow 0, n$ and $\eta$ can be regarded as constant along the channel and $\sigma\left(S_{V}\right) / S_{V} \rightarrow 1 / \sqrt{N_{\mathrm{t}} k T L_{\mathrm{eff}} W_{\mathrm{eff}} / \gamma} \equiv\left(\sigma\left(S_{V}\right) / S_{V}\right)_{0}$ as expected. 
As $V_{\mathrm{ds}}$ increases, $n$ near the drain decreases and $\eta$ increases from the source to the drain, which leads to the increase of the first term of Eq. (2). The fluctuation calculated with Eq. (2) is plotted in Figs. 5, 6. The carrier concentration $n$ in the variable $\eta$ is calculated from $n(x)=C_{\mathrm{ox}}\left(V_{\mathrm{gs}}-V_{\mathrm{th}}-V(x)\right) / q$, where the channel potential $V(x)$ is obtained from the drain current formula based on the gradual channel model with velocity saturation effect taken into account (Ko 1989). The simulated data explain the drain and gate bias dependence of the noise fluctuation. As $V_{\mathrm{gs}}$ increases, the $\eta$ distribution approaches the uniform one shown in Fig. 7, and the noise fluctuation given by Eq. (2) is reduced.

\section{Conclusion}

Drain and gate bias dependent fluctuations of flicker noise of MOSFETs have been explained in terms of carrier concentration distributions in a MOSFET channel. The fluctuation in the saturation region is $2-2.5$ times that in the linear region.

\section{References}

Brederlow, R., Weber, W., Landsiedel, D. S., Thewes, R. (1999): Fluctuations of the Low Frequency Noise of MOS Transistors and their Modeling in Analog and RFCircuits. International Electron Devices Meeting Tech. Dig. pp. 159-162

Gray, P. R., Meyer, R. G. (1993): Analysis and Design of Analog Integrated Circuits. John Wily \& Sons, New York

Hajimiri, A., Lee, T. H. (1998): A General Theory of Phase Noise in Electrical Oscillators. IEEE J. Solid-State Circ. 33: pp. 179-194

Hung, K. K., Ko, P. K., Hu, C., Cheng, Y. C. (1990): A Unified Model for the Flicker Noise in Metal-Oxide-Semiconductor Field-Effect Transistors. IEEE Trans. Electron Devices 37: pp. 654-665

Ko, P. K. (1989): Approaches to Scaling. In: Einspruch, N. G., Gildenblat, G. S. (eds.): VLSI Electronics Microstructure Science. Academic Press, San Diego, pp. 1-37 (Advanced MOS Device Physics, vol. 18)

Pelgrom, M. J. M., Duinmaijer, A. C. J., Welbers, A. P. G. (1989): Matching Properties of MOS Transistors. IEEE J. Solid-State Circ. 24: pp. 1433-1440

Ziel, A. v. d., Park, H. S. (1979): A discrepancy in the elementary theory of MOSFET modeling. Appl. Phys. Lett. 35: pp. 942-944

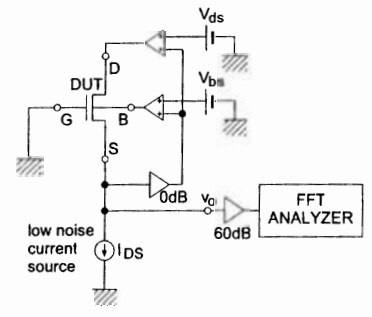

Fig. 1. Schematic of noise measurement setup.
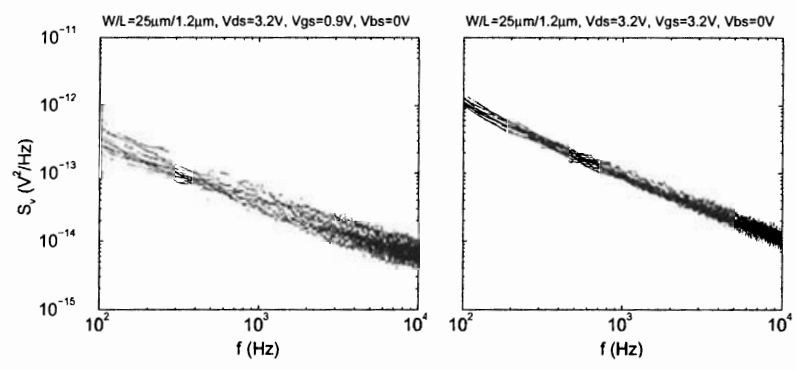

Fig. 2. Examples of measured input referred noise voltage spectrum of 35 samples for each bias condition. $V_{\mathrm{ds}}=3.2 \mathrm{~V}, V_{\mathrm{gs}}=0.9 \mathrm{~V}($ left $), 3.2 \mathrm{~V}$ (right). 


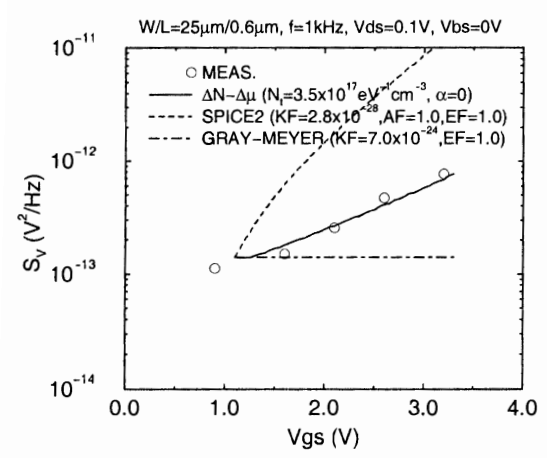

Fig. 3. The gate bias dependence of the input referred noise voltage.
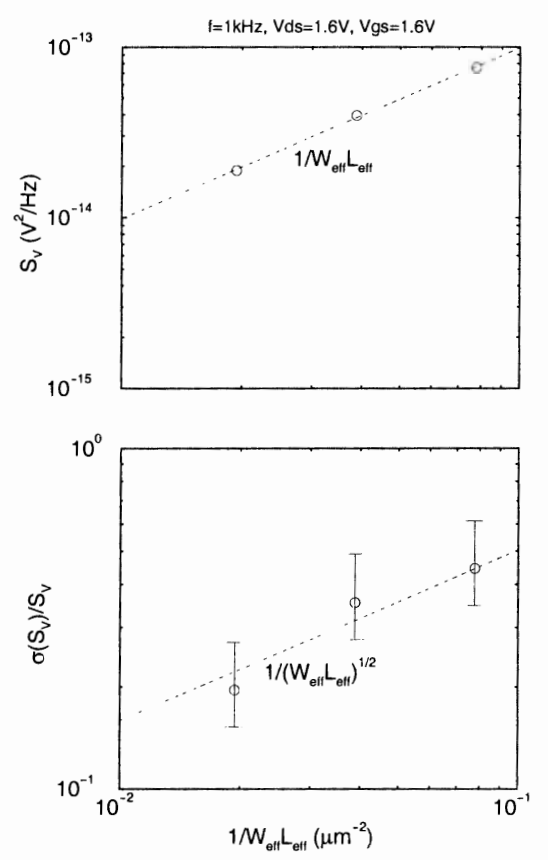

Fig. 4. The gate area dependence of the average (upper figure) and the standard deviation (lower figure) of the input referred noise voltage power. The error bars show confidence interval with the confidence coefficient of $98 \%$.

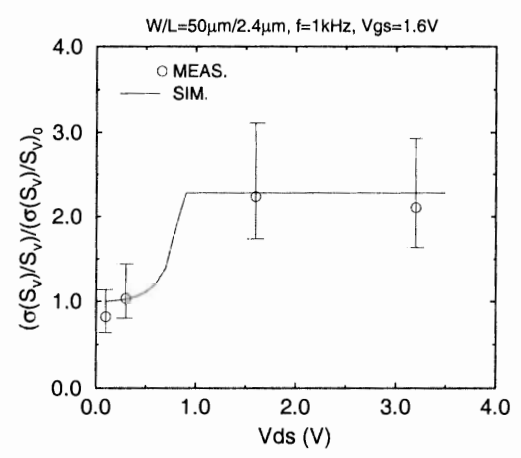

Fig. 5. The drain voltage dependence of the normalized standard deviation of the input referred noise voltage power. The measured data have been divided by $1 / \sqrt{N_{\mathrm{t}} k T L_{\text {eff }} W_{\text {eff }} / \gamma}$.

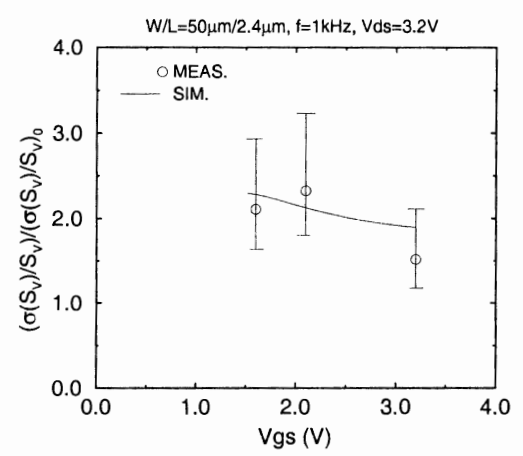

Fig. 6. The gate voltage dependence of the normalized standard deviation of the input referred noise voltage power.

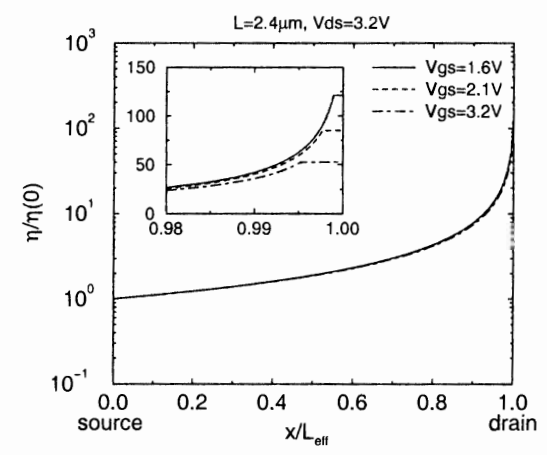

Fig. 7. The parameter $\eta$ as a function of position in the channel. 